\title{
III. \\ Die Rechtsgelehrten \\ Hans Jacob und Christian Jacob von Zwierlein (1699-1793). \\ Von
}

\author{
Herrn Archivar a. D. F. W. E. Roth \\ in Wiesbaden.
}

Ueber diese beiden Rechtsgelehrten ist wenig bekannt. Es ist mir gelungen, das reiche aber ungeordnete und einer gelegentlichen Zerstreuung ausgesetzte Archiv der Familie v. Zwierlein zu Geisenheim zu durchsuchen. Die nachstehenden Mittheilungen beruhen grösstentheils auf dieser Quelle.

Hans Jacob Zwirlein, wie er sich in Briefen und Drucksachen schrieb, ward am 9. Februar $1699 \mathrm{zu}$ Worms a. Rh. von reformirten Eltern geboren. Sein Vater war der von Frankfurt a. M. nach Worms übergesiedelte Handelsmann Johann Friedrich Zwirlein, seine Mutter Anna Maria Schäfferin, Tochter des Nicolaus Schaffer, Bürgers und Handelsmanns zu Nürnberg. Einer der Taufpathen des Hans Jacob Zwirlein war Johannes Zwirlein, ,des beständigen Regiments der Herrn Dreizehner" zu Worms Mitglied, welcher 1697 am 23. December im dreizehnten Colleg vorkommt ${ }^{1}$ ). Hans Jacob besuchte das Gymnasium zu Worms und studirte dann zu Giessen Rechtswissenschaft. Dort war Johann Georg Estor (geboren 1699) sein Studiengenosse und bildete sich $z$ wischen Beiden eine lebenslängliche Freundschaft. Zwirlein ward 1723 AdvocatSchreiber am Reichskammergericht zu Wetzlar, 1728 Licentiat der Rechte und 1730 Procurator am Reichskammergericht,

1) Moriz, Abhandlung vom Ursprung der Reichsstädte. 1756. S. 581. 
nachdem er eine dissertatio inauguralis de favore ultimarum voluntatum verfasst und im Druck veröffentlicht hatte. 1738 stand er mit Estor in Briefwechsel ${ }^{1}$ ) und lernte im gleichen Jahr den Reichshofrath H. Christ. von Senckenberg aus Frankfurt a. M. kennen, der ihn am rheingräflichen Hof zu Dhaun, wo er Rath war, einführte. Das Jahr 1742 brachte Zwirlein die Ernennung zum königlich grossbritannischen Hofrath und kurfürstlich Braunschweig-Lüneburgischen Hofrath. Beider Höfe Vertreter ward er zu dieser Zeit für deren Angelegenheiten am Reichskammergericht. 1743 sollte Zwirlein Lehrer des Völkerrechts zu Halle werden, erreichte aber seinen Zweck nicht, da Johann Jacob Schmauss die Stellung bekam. 1737 hatte Zwirlein die Elisabethe Dorothea Friderike Wahl (getauft 9. Juli $1718 \mathrm{zu}$ Wetzlar) geheirathet und scheint dadurch zu nicht unbedeutendem Besitz zu Wetzlar, durch seine Verbindungen mit Fürstenhöfen auch zu grossem Einkommen gelangt zu sein. Er strebte deshalb nach dem Adel und suchte in einer Zeitung nach einem Vermittler desselben. v. Senckenberg meldete sich auf diese Anzeige hin. Zwirlein betonte, er sei viele Jahre des Königs von Grossbritannien, des Prinzen von Oranien, des Markgrafen von Brandenburg fränkischer Linie, sowie anderer Fürsten Vertreter beim Reichskammergericht gewesen und habe sich durch die Herausgabe der Reichskammergerichtsordnung verdient gemacht. Er beabsichtige eine neue Ausgabe derselben und wolle solche dem Herrn von Brandau und dem Grafen von Cobenzl widmen, um deren; Vermittlung zu erreichen. Diese Vorschläge machte Zwirlein in einem Briefe an v. Senckenberg am 27. September 1751 von Wetzlar aus. Dieser versprach, als kaiserlicher Pfalzgraf Wappenbrief und Adelsdiplom zu beschaffen, brachte die Sache auch zu Wien ins Reine und strich die 350 Gulden Vermittelungsgeld ein. Am 24. Februar 1752 ertheilte der erwählte römische Kaiser Franz I. von Wien aus dem Johann Jacob Zwierlein, wie er jetzt hiess, ,was massen er nicht allein bey Unserm und des Reichs Cammer-Gericht schon viele Jahr hindurch als Procurator stehe, sondern auch bey dem königlich Gross-Brittanischen Hof sowohl als ver-

1) Estor, Auserlesene kleine Schriften XI, 566. Brief Joh. Jacobs au Estor, Jena den 25. September 1738. 
schiedenen Fürsten und Ständen des Reichs als Hof-Rath rühmlichst gedienet, undt endlich seine Gelehrtsamkeit und ausnehmende Erfahrung durch verschiedene in iure Camerali heraus gegebene Schrifften schon sattsam gezeiget habe", für ihn und dessen eheliche Leibeserben beiderlei Geschlechts den Reichsadelstand und das jetzige Familienwappen, einen grünen Schild mit golden und roth abwechselnd geschachtem Rechts-Schrägbalken versehen ${ }^{1}$ ). Devise ward: Durant virtute parata. Am gleichen Tage verlieh Kaiser Franz I. dem Hans Jacob von Zwierlein den Titel eines kaiserlichen Pfalzgrafen und Maria Theresia verehrte ,ihrem lieben Getreuen" Hans Jacob von $Z$ wierlein ein Paar silberne Schuhschnallen mit Brillanten und liess solche demselben mit einem höchst schmeichelhaften Schreiben zustellen. Die neue Ausgabe des Concepts der Reichskammergerichtsordnung erschien 1754 und zwar auf Grund einer Handschrift des 17. Jahrhunderts, welche v. Senckenberg dem Herausgeber geschenkt hatte. Diese Handschrift ist noch vorhanden. v. Senckenberg erhielt 1753 ein Geschenk von Rheinwein für die glückliche Erledigung der Sache, da er auch die Erhebung der Gattin v. Zwierleins in den erblichen Adelstand $\mathrm{zu}$ Wien fertig bringen sollte. Am 5. Januar 1753 versprach Kaiser Franz I. die Fertigung auch dieses Adelsbriefs. v. Zwierleins Gattin entstammte einem adeligen, aber herabgekommenen und deshalb den Adel nicht mehr führenden Geschlecht. Kaiser Franz I. ertheilte auf v. Senckenbergs Betreiben am 18. Mai 1753 der Susanna Dorothea geborenen Hubin von Gülchen, verwittibten Wabl, Mutter der Gattin Zwierleins, Adelsbrief nebst Wappen auf ältere Wappenbriefe hin gleichsam als Erneuerung solcher ${ }^{2}$ ). Zwierlein wohnte zu Wetzlar in dem Familienhofe am Oberthor. Am 15. September 1753 nahm ihn die Reichsritterschaft in ihren Verband auf, da er unterdessen von den Erben v. Buseck das Gut zu Winnerod, ferner zu Nauborn, Bubenrod und Lützellinden, alle in der Wetterau gelegen, käuflich erworben hatte. Er nannte sich fortan Erb- und Gerichtsherr zu Winnerod und Bubenrod. Seine Einkünfte waren grossartige. Die Vertretungen von mehr als einem Dutzend fürstlicher Höfe am bulle.

1) Orig. Diplom mit Silberbulle. - ${ }^{2}$ ) Orig. Adelsbrief mit Silber- 
Reichskammergericht und manche Geschäfte, welche Zwierlein ausserdem an Höfen besorgte, brachten grosse Summen ein. Am rheingräflichen Hof zu Dhaun und Kirburg, bei dem Fürsten von Oranien zu Dillenburg, zu Anhalt-Bernburg, wo er am 31. August 1754 Gebeimrath wurde, am Hof zu Neustrelitz, dessen Vertreter er am 6. Januar 1762, und am Lübeck'schen Hof, wo er am 13. November 1765 mit 300 Gulden Jahresgehalt angestellt ward, stand er wegen seiner Gewandtheit in hohem Ansehen. 1741 war er zu Göttingen Doctor der Rechte geworden. Mehrfach gab ihm der kaiserliche Hof seine $\mathrm{Zu}-$ friedenheit zu verstehen, 1754 verehrte ihm Kaiser Franz I. eine Sendung Ungarwein, der aber durch Versehen des Spediteurs zu Frankfurt a. M. verdarb und ausgeschüttet werden musste.

Aus der Ehe mit Elisabethe v. Wahl hatte Hans Jacob v. Zwierlein folgende Kinder:

1. Christian Jacob, geboren 1738 als ältestes Kind.

2. Johann Gottfrid, geboren 1739 zu Wetzlar, vermählt an Louise v. Buseck, ebenfalls Rechtsgelehrter, Salm-Salmischer Regierungsrath. Er war scheel und hatte deshalb den Namen „der scheele Zwierlein". Er starb zu Frankfurt a. M. und verfasste: Ordinis iuridici prodecanus Ludovicus Boemer viri generosissimi Victoris a Saussure equitis Lausannensis viri generosissimi Ioannes Godefredi Zwierlein Wetzlariensis viri praenobilissimi Ioannis Adami Meixneri Wormatiensis solemnia inauguralia indicit. Goettingae 1766. Quarto, 32 Seiten $\left.{ }^{1}\right)$, und: de ordine iudiciario ab austraegis observando. Goettingae 1765. Quarto ${ }^{2}$ ).

3. Friedrich Salentin, geboren 17. Januar 1747, gestorben als fürstlich Solms-Braunfels'scher Geheimer Rath und Regierungspräsident den 6 . October 1803 , vermählt an Caroline Albertine geborne v. Harling (geboren 22. October 1754, gestorben 17. April 1804). Beide Eheleute wurden in der Kirche zu Winnerod beerdigt, wo ihr Denkmal sich befindet.

4. Louise starb unvermählt.

5. Christiane, Gattin eines Schenk zu Schweinsberg.

1) Mainz, Stadtbibliothek. $-{ }^{2}$ ) v. Fahnenberg, Literatur des Reichskammergerichts, S. 259 und 158. - J. Jac. Moser, neueste Geschichte der Teutschen Staats-Rechts-Lehre. 1770. S. 190. 
Hans Jacob v. Zwierlein starb am 20. Juni 1772 früh vor 5 Uhr zu Winnerod an einem Kolikanfall im Alter von 73 Jahren und ward nach dem Winneroder Kirchenbuch am 23. Juni 1772 früh Morgens in aller Stille um 6 Uhr in das von ihm erbaute Erbbegräbniss der Kirche, um die er sich durch ihre Herstellung und Guss einer Glocke verdient gemacht, beigesetzt. Sein Grabstein erbielt die Inschrift: Ioannes Iacobus de Zwierlein I. U. D. Dominus in Winneroda et Bieberoda (!) comes Palatinus Caesareus, principis Anhaltini Bernhurgensis consiliarius intimus actualis, camerae imperialis procurator, natus $9^{\text {a }} \mathrm{Feb}$. 1699 Wormatiae, mortuus $20^{2}$ Iunii 1772 Winnerodae. Seine Gattin Elisabeth starb zu Wetzlar den 14. Februar 1797 und liegt neben ihrem Gatten zu Winnerod begraben. Sie erhielt diese Inschrift: Elisabetha Dorothea Friederica Baronissa de Zwierlein, nata ex familia de Wahl nominata Hubin de Gülchen baptizata 9 Iulii 1718 Wetzlariae, mortua ibidem 14 Febr. 1797. Hans Jacobs v. Zwierlein Bildniss in Oel gemalt (Brustbild in rothem Mantel und gestickter Weste) hängt zu Geisenheim im Familienhof, ein Stahlstich desselben Bildes ist einem Exemplar des Concepts der Kammergerichtsordnung von 1743 vorgebunden, welches zu Geisenheim vorhanden. Seine reiche Bibliothek mit den eigenhändigen Einträgen: Ex libris Ioann. Iacobi Zuirlein etc. ist noch zu Geisenlıeim erhalten. Daselbst befinden sich auch noch seine Haushaltungsbücher, aber aus seinem Briefwechsel Weniges.

Christian Jacob v. Zwierlein war geboren zu Wetzlar 1738, studirte zu Göttingen Rechtswissenschaft und ward 1757 Doctor beider Rechte. Er ward Assessor am Reichskammergericht und Vertreter zahlreicher Fürstenhöfe. Kammerrichter v. Gülich war sein besonderer Gönner. Durch seinen Bruder Johann Godfrid ward er am Salm-Salmischen Hof eingefuibrt und wurde dessen Vertreter in der schwebenden Erbfolgesache. 1777 wurde er wirklicher Geheimrath. Christian Jacob verlobte sich am 12. November 1765 mit Christine Friderike, Tochter des Johann Friedrich Erasmus v. Hopfer und der Christine Elisabeth geborenen Wolf zu Tübingen. Die Hochzeit fand am 2. Mai 1766 zu Wetzlar statt. v. Zwierlein erhielt durch seine Frau 10000 Gulden Brautschatz, welche von deren Vater am 13. Mai 1766 auf das Gut Langsdorf in der Wetterau angewiesen 
und für 1767500 Gulden Rente aus diesem Gut verschrieben wurden. Am 27. October 1781 sicherte v. Hopfer dem Herrn v. Zwierlein $z \mathfrak{u}$, wenn derselbe das Langsdorfer Gut nicht bei seinen Lebzeiten für 40000 Gulden käuflich erwerbe, solle dasselbe mit allem Inventar bei der Erbtheilung nur für diese Summe angerechnet werden. Am 7. März 1782 kaufte v. Zwierlein das Langsdorfer Gut für diese Summe und bezahlte dieselbe bis zum 11. April 1783 baar aus. Ausserdem erhielt er die Anwartschaft auf einen Theil des Gutes Hopfers zu Unterrixingen und zu Geisenheim im Rheingau. Nur letzteres kam später an die Familie. Am 4. November 1772 ward Christian Jacobs Schwiegermutter Christine Elisabeth vom Kaiser Joseph II. in den Adelstand als Wolf von Schlimmenberg erhoben und am 22. November 1788 das Adelsdiplom für die Mutter Christian Jacobs erneuert und bestätigt. Als 1789 der bekannte Lütticher Aufstand gegen den Fürstbischof von Lüttich ausbrach, spielte v. Zwierlein eine sehr hervorragende Rolle als Vermittler und gab auch eine hierauf bezügliche Druckschrift heraus. Am 15. Mai 1788 ward er Vertreter für Karl August, Herzog von Sachsen, am Reichskammergericht. Christian Jacob starb auf seinem Gute Langsdorf in der Wetterau am 11. August 1793 in Folge eines Schlaganfalls und ward in der dortigen Kirche begraben. Am 26. August 1793 liess Herzog Ernst von Sachsen den Empfang der Todesnachricht anzeigen und sprach dessen Sohn, dem Hans Carl v. Zwierlein, Reichskammergerichtsprocurator zu Wetzlar, sein Beileid aus, indem er solchen zugleich zu seinem Bevollmächtigten am Reichskammergericht ernannte. Aus der Ehe mit Christine v. Hopfer war Hans Carl hervorgegangen, welcher ebenfalls die Rechte studirte und in Allem Nachfolger seines Vaters ward. Am 3. Februar 1790 ernannte Herzog Adolf Friedrich von Mecklenburg den Hans Carl zum Nachfolger seines Vaters als Vertreter am Reichskammergericht und am 1. März 1793 bestätigte denselben als solchen der Fürst von Nassau-Saarbrücken. Christian Jacob hat hohe Bedeutung als juristischer Schriftsteller, als scharfsinniger Deducent; seine Arbeiten in dieser Beziehung zeichnen sich namentlich durch den Abdruck werthvoller Urkunden aus und sind immer noch historische Fundgruben für den Forscher. 
Dieselben sind in einem Sammelbande zu Geisenheim fast alle vereinigt.

\author{
Schriften.
}

\title{
1. Hans Jacob von Zwierlein.
}

1. Dissertatio inauguralis de favore ultimarum voluntatum. $0 . \mathrm{J}$. (1736.) Darmstadt, Hofb.

2. Concept der auf kayserlichen und des reichs befehl im Jahr 1613 verbesserten Cammergerichts ordnung, auf das sorgfältigste übersehen und mit anmerkungen vermehrt von Johann Jacob Zwirlein kônigl. Grosbritann. und churf. Braunschw. Lüneb. Hofrath auch des kayserl. cammergerichts advocaten und procuratorn. Giessen. 1744. Octavo, $600+18+116$ Seiten. Das Vorwort endet: Wetalar den 2 December 1743. Johann Jacob Zwirlein Dr. - In meinem Besitz. Darmstadt, Hofb.

3. Beylagen zu den Estorischen anfangsgründen des gemeinen und reichsprocesses, worin ein sehr richtiger abdruck des concepts der cammergerichtsordnung und jüngsten reichsabschieds ingleichen der Ludolffischen anmerckungen über ersteres enthalten. Haupttitel: Concept etc. wie oben. - Franckfurt a. Mayn 1753. Vorrede datirt: Wetzlar den 1 September 1752. Quarto. $16+392+31$ Seiten. In meinem Besitz, Darmstadt Hofb.

4. Eine Ausgabe des ius camerale des Melchior v. Ludolf ${ }^{2}$ ).

\section{Christian Jacob von Zwierlein.}

1. Dissertatio prima de litteris requisitorialibus ex usu Romanorum antiquiori et recentiori etc. Goettingen. 1758. Quarto. $8+55$ Seiten. Mainz St. B., Darmstadt Hofb.

2. Dissertatio secunda de litteris requisitorialibus ex usu Germanorum antiquiori et recentiori etc. Goettingen. 1758. Quarto. $4+78$ Seiten. Ebenda.

3. Geschichts-Erzählung in Sachen des Klosters Banz wider die Herzoglich-Sachsen-Hildburghausische Regierung etc. 1762. 0. O. Folio. $12+88$ Seiten. Sammelhand der Schriften Chr. J. v. Zwierlein zu Geisenheim.

4. Vollständiger status causae in Sachen der Schwarz- und Schönfärber zu Frankfurt am Mayn wider Lewin Christian Wegener und den Löblichen Schöffen-Rath daselbst. 1766. Folio. 32 Seiten. Sammelband.

5. Vermischte Briefe und Abhandlungen über die Verbesserung des Justizwesens am Kammergerichte, mit patriotischer Freimüthigkeit entworfen. Berlin. 1767. Octavo. 3 Theile. Darmstadt Hofb.

6. Vermehrte Beiträge zur Verbesserung des Justizwesens am Cammergericht. Frankfurt a. M. 1768-69. Octavo. 3 Theile. Darmstadt Hofb. Neuauflage des vorigen Werks.

1) Fahnenberg, Literatur S. 14 n. 3. 
7. Abgedrungene Vorstellung von Seiten des regierenden Herrn Fürstens Ludwig Carl Ottens zu Salm-Salm das von seinem jüngeren Herrn Bruder, Prinz Maximilian beeinträchtigte Erstgeburths und Siz und Stimmrecht auf dem Reichstag, wie auch den von höchstbesagten Herrn Prinzen vorsezlich erregten Jurisdictions-Streit zwischen beiden Reichsgerichten betreffend etc. 1770. 0.0. Folio, $10+12+88+112$ Seiten. Sammelband, Darmstadt Hofb.

8. Rettung der Rechte des Erstgebohrnen in dem Fürstlichen Hause Salm-Salm welche die Lehensfolge der Geistlichen nach teutschen Gesezzen und Gewohnheiten erweiset zur ferneren Aufklärung verschiedener an dem höchstpreislichen Kaiserlichen Cammergericht rechtshängiger und von da an den höchstpreislichen Kaiserlichen Reichshofrath gezogen werden wollender Processe etc. 1771. Folio, $10+148+50$ Seiten. Sammelband, Darmstadt Hofb.

9. Peregrinus ex comitiis tum generalibus tum particularibus provinciae Leodiensis exsors dissertatio dilucidationi litis inter status tertii sine popularis ordinis in supremo camerae imperialis tribunali agitatae, inserviens in causa Grouwels et cons. contra Michiels et cons. appellationis. Wetzlar. 1771. Folio, 62 Seiten. Sammelband.

10. Historisch-diplomatische Abhandlung von denen Landesherrlichen Gerechtsamen des Hochfürstlichen Hauses Brandenburg über den Marktflecken Fürth etc. O. O. (Ansbach). 1771. Folio, 144+162 Seiten. Sammelband. Vgl. v. Zwierlein, Nebenstunden S. 234 Anm. 2.

11. Unterthänigste Supplication und Bitte Pro clementissime concedenda restitutione in integr. adversus sententiam d. 7. September a. c. publicatam iuncta causalium deductione in Sachen Wied-Runkel contra Nassau-Hadamar etc. Folio, $14+2$ Seiten. Sammelband.

12. Pro memoria in Sachen des Herrn Grafens von Kielmansegge und Consorten wider die Universität zu Kiel praet. Appellationis. Folio, 6 Seiten. O. J. (1773). Sammelband.

13. Rechtliches Gutachten über den in unentschiedenen Rechten schwebenden Process in Sachen die Freiherrn von Sickingen wider die Freiherrn von Saint-André Dec. Appellationis nune petitae restitutionis in integrum iuncta litis denunciatione nebst einem vollständigen Auszug der Acten. 1774. Folio, $6+43+35$ Seiten. Sammelband.

14. An eine Höchstansehnliche Kayserliche Commission und Hochverordnete Reichs-Visitations Deputation, gemüssigte Bitte um Promotoriales an das Höchstpreissliche Reichs-Cammergericht in Sachen des regierenden Herrn Marggraven zu Baden Hochfürstlichen Durchlaucht contra das Gotteshauss Frauenalb etc. 1775. Folio, 4 Seiten. Sammelband.

15. Erörterung der Frage, ob Vater und Sohn oder sonst zwei nahe Verwandte zu gleicher Zeit auf denen Richterstühlen des Kaiserlichen Reichs Cammer-Gerichts sizzen können nach denen Gesezzen und dem Herkommen. 1775. Folio, $2+16$ Seiten. Sammelband.

16. Erörterung bejderseitiger Rechtsgründe in Sachen des Herrn Grafen von der Schulenburg contra Braunschweig-Wolfenbüttel praetensae citationis. etc. 1778. Folio, $6+56+6$ Seiten. Sammelband. 
17. Rechtfertigung des Landesfürstlichen Verfahrens beim Kirchheimer Tumult etc. Wetzlar. 1778. Folio, $2+83+56$ Seiten. Vgl. v. Zwierlein, Nebenstunden S. 204 Note ${ }^{*}$ ). Sammelband.

18. Ghristian Jacob von Zwierleins Hochfürstl. Anhalt-Bernburgischen wirklichen Geheimdenraths und des kaiserlichen Kammergerichts Procurators Nebenstunden. I. Theil. Giessen. 1778. Octavo, $16+285$ Seiten. Mehr erschien nicht. In mejnem Besitz, Darmstadt Hofb.

19. Zwey Fragen aus dem Lehenrecht. 0.0. u. J. (1778). Octavo. Darmstadt Hofb.

20. Examen litis inter baronem de Weichs ab una et baronem de Collenbach ab altera parte in duobus supremis imperii trihunalibus puncto praepositurae in Hansinne agitatae. Wetzlar. 1779. Folio, $4+32$ Seiten. Sammelhand.

21. Erörterung beiderseitiger Rechtsgründe in Sachen derer Herrn Vormünder der Demoiselle Antoinette Carstens modo ihr selhst Appellanten wider die Herrn Executoren des von weil. Herrn Senator Bōtefeur errichteten Testaments etc. 1779. Folio, $88+12$ Seiten. Sammelhand.

22. Ad s. R. i. comitia generalia querelae legati Leodiensis delatae adversus iudicata consilii imperialis aulici in causa baronis de Weichs ab una contra baronem de Collenbach atque Bernisium at altera parte etc. Leodii. 1780. Folio, 6 Seiten. Sammelband.

23. Examen litis inter baronem de Weichs ab una et barones de Collenbach atque dominum cardinalem de Bernis eiusque nepotem de Pierre de Bernis ab altera parte etc. 0. 0. 1780. Folio. $10+62+98$ Seiten. Sammelband. Darmstadt Hofb.

24. Pro memoria in Sachen des Herrn Reichsgrafens von Rice gegen den Bürger Bertrand zu Spa und den Herrn Official zu Lüttich einen unrechtmässigen Arrest betr. 1780. Folio, 13 Seiten. Sammelhand.

25. Phlichtmässiges Gutachten über die in Berathschlagung stehende Materien von der Reichs-Cammergerichtlichen Justiz-Verbesserung. Wetzlar. 1788. Octavo. Darmstadt Hofb.

26. Kurze Uehersicht des Lütticher Aufruhrs vom Jahr 1789. O. O. (Wetzlar). 1789. Folio. Darmstadt Hofb.

26a. Memoire instructif sur la revolte Liègeoise. Wetzlar. 1789. Quarto. Darmstadt Hofb.

27. Beleuchtung der Bemerkungen, welche iher die Amnestie vom 20. Oct. 1791 gedruckt worden sind. Wetzlar. 1791. Octavo. Darmstadt Hofl.

Ohne Jahresangabe.

1. Pro memoria in causa Theresiae de Hallebaye, dotalitiae de Gradii contra de Harenne eiusque uxorem Deodatam Dethier dotalitiam Iohannis Caroli de Gradii appell. primae. O. O. Quarto. 4 Seiten. Sammelband. 
114 F.W. E. Roth, Hans Jacob u. Ghristian Jacob v. Zwierlein.

2. Pro memoria in causa dominae dotalitiae de Gradii de Croenendael contra dominum advocatum de Harenne dominamque eins uxorem, appellationis secundae. Quarto, 4 Seiten. O. 0 . Sammelhand.

3. In causa domini comitis de Pontii novi monasterii abbatis eiusque nobilis monasterii contra Hubertum Iosephum Corbeau suppl. pro processibus appellationis maturationem clementissimam decreti humillime rogat Dr. de Zwierlein. Folio, 4 Seiten. O. 0. Sammelband. Betrifft das Kloster Neufmostier.

4. Unterthänigste Berichtserstattung Bürgermeister und Rath der Stadt Bremen in Sachen des Schuhmacheramts zu Bremen wider das Loligerberamt daselbst. Folio, 39 Seiten. O. 0. Sammelband.

5. Unterthänigste Supplication und Bitte pro clementissime decernendo mandato poenali s. c. de non contraveniendo R. I. de 1544 paci religiosae atque Westphalicae sed ad ipsam litteram pacis Westphalicae Art.V. $\$ 47$. restituendo monasterii Beselich etc. Folio, 28 Seiten. Sammelband.

6. Pro memoria in Sachen Reinhard Witkens wider den löblichen Magistrat zu Bremen praetensae suppl. pro citat. super nullis. Folio, 6 Seiten. Sammelband. Wiesbaden L. Bibl. (1775).

7. Geschichtserzehlung und vollständige Prüfung beiderseitiger Rechtsgründe in Sachen des Klosters Riechenberg wider die Herren Deputirte Hochfürstlicher Regierung und der Landstände des Hochstifts Hildeshein Mandati de cassando iniustas contrib. G. C. Folio, 14 Seiten. Sammelband ${ }^{\mathbf{1}}$.

Nebstdem beschäftigte sich Christian Jacob von Zwierlein mit Geschützwesen, Festungshaı und Bauwesen überhaupt. Das Familienarchiv zu Geisenheim besitzt: Collegia pirothechniae, architect. militaris et civilis conscripta a Christ. Iacobo de Zwirl. Hs. Quarto, mit vielen höchst feinen Tuschzeichnungen. Das Mspt. behandelt Geschützwesen, Geschützladungen, Festungsbau nach Vauban, Säulenstellungen der Griechen und Italiener, sowie Hausbau.

1) Joh. Jac. Moser, neueste Geschichte der Teutschen Staats-RechtsLehre S. 191, nennt noch als von Chr. Jac. v. Zwierlein verfasst: modus et norma instituendi et prosequendi processum austraegarum. 